\title{
Neutron reflectometry and hard X-ray photoelectron spectroscopy study of the vertical segregation of PCBM in organic solar cells
}

\author{
Antonio Urbina ${ }^{\mathrm{a}}$, José Abad ${ }^{\mathrm{a}}$, Antonio J. Fernández Romero ${ }^{\mathrm{a}}$, Jesús S. Lacasa ${ }^{\mathrm{b}}$, Jaime Colchero ${ }^{\mathrm{b}}$, \\ Juan F. González ${ }^{c}$, Juan Rubio-Zuazo ${ }^{\text {d, e, Germán R. Castro }}{ }^{\text {d, e }}$, Philipp Gutfreund ${ }^{f}$ \\ ${ }^{a}$ Advanced Materials for Production and Storing of Energy, Technical University of Cartagena, Plaza del Hospital 1, 30202 Cartagena, Spain \\ ${ }^{\mathrm{b}}$ Departament of Physics, Instituto Universitario de Investigación en Óptica y Nanofísica (IUIOyN), University of Murcia, Campus de Espinardo, 30100 Murcia, Spain \\ ${ }^{c}$ Department of Biomedical Sciences, Malmö University, 20506 Malmö, Skåne, Sweden \\ d SpLine CRG BM25 Beamline, European Synchrotron Radiation Facility, 71 Avenue des Martys, 38000 Grenoble, France \\ ${ }^{\mathrm{e}}$ Instituto de Ciencia de Materiales de Madrid, Consejo Superior de Investigaciones Científicas (ICMM-CSIC), 28049 Madrid, Spain \\ ${ }^{\mathrm{f}}$ Institut Laue-Langevin, 38042 Grenoble Cedex, France
}

\section{A R T I C L E I N F O}

\section{Keywords:}

Organic solar cells

Neutron reflectometry

Hard X-ray photoelectron spectroscopy

Conjugated polymers

P3HT:PCBM

\begin{abstract}
A B S T R A C T
The composition of the active layer of a benchmark functional glass/ITO/[P3HT:PCBM][1.1:1]/Al organic solar cell has been studied by neutron reflectometry (NR) and hard X-ray photoelectron spectroscopy (HAXPES). Thermal annealing was performed in several steps and NR and HAXPES were recorded for every temperature. By fitting the NR results to a model composed of several layers, the scattering length density (SLD) distribution through the sample was obtained, and from this SLD profile, the evolution of the composition of the active layer as a function of temperature was established. For the outer layers, HAXPES results confirm the composition evolution. The results show that PCBM tends to segregate reducing the initial concentration of PCBM in the central part of the active layer and increasing its concentration towards both interfaces. The effect of the $\mathrm{Al}$ electrode as studied by HAXPES on the nearest zone of the active layer (up to $50 \mathrm{~nm}$ ) is to stabilize the P3HT depletion in this area, an effect which is not affected by thermal annealing.
\end{abstract}

\section{Introduction}

Emerging photovoltaic technologies are currently being developed. Plastic solar cells are within the most promising proposals, which could deliver "solar electricity" at lower cost than other conventional technologies and therefore reach grid-parity in a shorter term $[1,2]$. The active layer of the device is made of a blend of two organic materials, one which acts as electron acceptor ( $n$-type) and the other as electron donor ( $p$-type): their mixture creates a distributed "bulk heterojunction (BHJ)" $[3,4]$. Usually the electron donor partner in the BHJ is a conjugated polymer and the electron acceptor a fullerene derivative. The bench mark device has been the one including a poly-3-hexyl-thiophene/ methano-fullerene [P3HT:PCBM] active layer embedded between different electrodes and in most cases including hole or electron injecting layers that were deposited from solution and subsequently annealed $[5,6]$. Although limited in power conversion efficiency (best devices around 5\%, most often reported around 3\%), the P3HT has al- lowed the exploration of different device architectures, of which the standard and the inverted architectures played with the HOMO/LUMO levels of the polymers and the work function of the electrodes to create the adequate drift/diffusion equilibrium throughout the device in order to extract the carriers before the long-lived excitons are recombined. More recently, tandem devices including different conjugated polymers, ideally with lower band gap have been included in the device architecture and improved power conversion efficiencies achieving beyond 10\% [7-9]. Also, the use of non-fullerene acceptors in organic solar cells, providing power conversion efficiencies above $13 \%$ opens a promising route for next generation OSCs where fullerene is replaced by perylene-diimides or fused aromatic cores [10]. In the simplified standard architecture of a solar cell, the photogenerated electrons should be transferred to the aluminium electrode, whereas in the inverted architecture the electrons should be transferred to the ITO electrode; in both cases, the active layer, composed of a blend of P3HT and PCBM will benefit from a composition gradient of opposed orientation: increment of PCBM towards the dark side (Aluminium electrode) and

Email address: antonio.urbina@upct.es (A. Urbina) 
depletion of PCBM towards the illuminated side (PEDOT:PSS/ITO electrode) in the case of the standard architecture and the opposite in the case of inverted architecture. Therefore, the structure at the nanoscale within the active layer will determine the optoelectronic performance of the macroscopic devices. Besides, the influence of the compositional gradient may have an impact on stability, since the inverted architecture usually presents larger lifetimes [11-14]. The presence of a top electrode may have an effect both in the internal structure of the active layer and in the stability and therefore lifetime of the solar cell; more recently, besides standard $\mathrm{Al}$ (or Ag) electrodes, graphene layers are being incorporated to the structure of the solar cells with the purpose of increasing the lifetime and power conversion efficiency of the devices [15].

The understanding of self-organization processes of the blend when cast from solution (spin-cast, spray coated or slot-dye coated) together with the influence of post-casting annealing is necessary in order to design a manufacturing process which delivers the optimum nanostructure of the active layer with better power conversion efficiency and lifetime. This process will have an impact in the devices manufacturing cost and, taking into consideration the lifetime, also on the levelized cost of the produced energy [16].

The PCBM tends to aggregate and therefore destroy the path needed for $n$-type carrier transport; furthermore, a vertical segregation which creates a depth gradient of PCBM composition is also observed. A big research effort is currently devoted to understand the dynamical processes which drive such segregation. Several experimental tools have been used to measure the internal structure of the different layers comprising an organic solar cell, of which X-ray diffraction to measure the crystallinity of polymers and blends $[17,18]$ and also quasielastic neutron scattering to study the molecular dynamics of P3HT both in solution and in thin films $[19,20]$ are probably the most common. In this article the focus will be on neutron reflectometry and hard X-ray photoelectron spectroscopy, techniques which provide compositional gradients of buried layers with nanometric resolution.

The pioneering work by Björström et al. using dynamic secondary ion mass spectrometry (SIMS) demonstrated depth profiles showing composition waves that were caused by surface directed phase separation during spin-coating of APFO-3:PCBM samples [21]. Then, ellipsometry provided robust experimental results regarding compositional gradient; for example, Campoy [22] presented a work in which the influence of annealing was demonstrated on a P3HT:PCBM blend cast on a quartz substrate: before annealing, the PCBM content, which presents a constant reduction from a high $75 \%$ volume in the quartz side, is reduced to a low $15 \%$ volume immediately before the air surface and finally presents a $50 \%$ volume contribution at the surface itself, indicating protrusion of the PCBM. Upon annealing, this profile changes and the protrusions are reduced to around $25 \%$ volume while the composition immediately below the surface is increased to $50 \%$ volume, generating a "compositional inversion" with respect to the sample before thermal annealing. Also using ellipsometry and near-edge X-ray absorption fine structure spectroscopy (NEXAFS), changes in the hole transporting layer (HTL) are shown to significantly affect the BHJ interfacial segregation at the buried interface near the HTL while the composition near the free surface (air) of the BHJ is unaffected, the effect on power conversion efficiency is low despite this change in structure [23]. Additionally, there are indications that the increase of the annealing time contributed to a significant increase of the P3HT crystallinity at the top regions of the blend films [24], where the enrichment of P3HT also prevents the formation of PCBM clusters [25]. These results were later complemented by several groups using different techniques such as SIMS [26], X-ray photoelectron spectroscopy (XPS) [27] and Auger spectroscopy [28].

The technique that provides more detailed data on vertical composition is neutron reflectometry. For example, the experiment performed by Parnell et al. [29] with P3HT(protonated):PCBM(deuterated) blend spin cast on a Si wafer, in which the influence of solvent and thermal annealing was studied showing that PCBM tends to increase its \% volume contribution in both surfaces of the blend upon both kinds of annealing, but always presenting a higher concentration in the blend surface in contact with the Si substrate. Another result was obtained by Kiel et al. [30,31] with a fully protonated sample (also on a Si substrate): the air surface is dominated by P3HT before and after thermal annealing, although immediately under this surface the PCBM content is higher, up to a $80 \%$ volume, which is then reduced to a $50 \%$ volume fraction more or less constant throughout the blend volume until it reaches the Si-surface where it is increased again to almost $80 \%$ volume, the annealing displaces the peak towards the interior of the sample and increases slightly the PCBM concentration at the air surface from naught to a $21 \%$ volume. In summary, all experiments concerning compositional gradients point to similar pictures, with small differences regarding the thermal annealing process.

More recently, other groups have extended the application of the neutron reflectometry technique to study the profile of other blends used for active layers of solar cells, such as [PCDTBT:PC $\left.{ }_{71} \mathrm{BM}\right]$ [32]. This work showed that larger molecular weights lead to an increase in the conjugated polymer enrichment layer thickness at the anode interface; this evolution of composition improves the efficiency up to a limiting point where the polymer solubility causes a reduction of the PCDTBT concentration in the active layer.

In this article, we present a detailed study by neutron reflectometry (NR) and hard X-ray photoelectron spectroscopy (HAXPES) of the compositional gradient evolution upon thermal annealing of thin films which are building blocks of organic solar cells (i.e. using glass/ITO as electrode) and the full operating solar cell. Furthermore, annealing with and without back electrode ( $\mathrm{Al}$ ) has been performed and tested. In the following sections, the samples preparation and methodological details of experimental procedure and data fitting are described; then the results of NR and HAXPES are discussed; and finally we present the conclusions of the study.

\section{Samples and methods}

\subsection{Sample preparation and $I-V$ characterization}

For the fabrication of standard architecture organic solar cells, Indium Tin Oxide (ITO) $(20 \times 20 \times 1.1 \mathrm{~mm}$; Delta Technologies 4-8 $\Omega$ /square) coated glass slides were used as transparent substrates. Acetone (Sigma-Aldrich, purity 99.5\%), Isopropanol (Panreac, purity 99.5\%) and deionized water (Type $1,18.2 \mathrm{M} \Omega \mathrm{cm}$ ) were used for cleaning. Active layers were made up with a mixture of P3HT (poly-3-hexylthiophene) (Sigma-Aldrich, regioregular, purity 99.995\%) and PCBM (phenyl-C61-butyric acid methyl ester) (Solenne, purity 99\%) in a 1.1:1 wt ratio from a dichlorobenzene (Sigma Aldrich, purity 99\%) solution. The thickness of the active layer is of the order of $200 \mathrm{~nm}$ (initially estimated, later measured experimentally in detail by NR). Samples measured by NR does not include additional layers (neither PEDOT:PSS nor Al electrode) and therefore they are not functional devices.

The solar cell for HAXPES experiments includes a PEDOT:PSS and a capping $\mathrm{Al}$ top electrode $(38 \mathrm{~nm})$ which is a complete standard architecture organic solar cell: glass/ITO/PEDOT:PSS/[P3HT:PCBM]/Al. Therefore, PEDOT:PSS (poly-3,4-ethylene-dioxy-thiophene-polystyrene sulfonate) (Sigma-Aldrich 1.3\%wt water dispersion) was used as a hole injection layer in the solar cells tested in the HAXPES experiments, while those tested in the NR experiments are PEDOT:PSS free. PEDOT:PSS was filtered and spun coated on the cleaned substrate at $1800 \mathrm{rpm}$ before annealing it at $395 \mathrm{~K}$ for $10 \mathrm{~min}$ in order to remove water remains. Aluminium (Goodfellow, $\varnothing 0.25 \mathrm{~mm}$, and purity 
99.5\%) was used for thermal evaporation of "back" electrode contacts at $10^{-5}$ mbar pressures. Except for the metal contact deposition, all fabrication processes were carried out in air.

As a summary: samples reproducing partial or complete standard architecture of organic solar cells have been characterized by NR and HAXPES. The samples are: i) ITO on silica fused glass substrate (only NR experiments); ii) [P3HT:PCBM] [1.1:1] spin cast on glass/ITO substrate (NR and HAXPES experiments) and iii) solar cells with PEDOT:PSS hole injecting layer and $\mathrm{Al}$ electrode on top (only for HAXPES experiments). The samples represent the sequential step in the manufacturing process of a functional solar cell; only auxiliary hole or electron injecting layers have been omitted, these layers are not required for the cell to be operative, but are important to improve the cell power conversion efficiency. Nevertheless, neutron reflectometry experiment on structures that are part of solar cells provides information on processes which are closer to real production steps in comparison with previous experiments performed on ideal layers casted on substrates that are not used for solar cell manufacture. Also, since HAXPES is probing depths up to a maximum of $50 \mathrm{~nm}$ (see description of the method) the presence of PEDOT:PSS is not detected and does not affect the upper part of the active layer (in contact with air or with the Al electrode).

A Sun simulator AM1.5 (ABET Technologies Sun2000), a programmable voltage source (KEITHLEY 230) to make a voltage sweep and an electrometer (KEITHLEY 6514) have been used for measuring I-V curves of the organic solar cell. I-V characterization of the devices was made, resulting in reproducible power conversion efficiency (PCE) in a range between $1.5 \%$ and $3 \%$ with some best devices showing efficiencies up to $3.1 \%$ and most of devices showing efficiencies around $2.3 \%$. In particular the most reproducible ones where used for the HAXPES experiment, and the I-V curve of the sample is included as an insert in Fig. 7 (right). The low filling factor, in all cases below $50 \%$, is an indication of the problems for charge collection for these simple devices where only PEDOT:PSS has been used as hole injecting layer but no other electron or hole transport layers have been included between the active layer and the electrodes.

\subsection{Experimental methods: neutron reflectometry and HAXPES}

The calculated Neutron Scattering Length Densities (SLD) for P3HT and PCBM are respectively $6.72 \times 10^{-7} \AA^{-2}$ and $4.34 \times 10^{-6} \AA^{-2}$, providing enough scattering contrast to perform the experiments without the need of selective deuteration. The different samples measured correspond to the different steps in the fabrication procedure of a full operating organic solar cell with standard structure (typical size of samples for NR $2 \mathrm{~cm} \times 2 \mathrm{~cm}$ ). Besides, for all the samples, an annealing process between two NR measurements has been applied (in-situ, from room temperature to up to $415 \mathrm{~K}$ ), in order to analyse the influence of thermal annealing in the vertical diffusion of PCBM within the blends.

Neutron reflectometry is a technique which benefits from the interference patterns created by the reflection of the neutron wave at different interfaces as explained in more detail in Annexure I. The refraction and reflection interference patterns created by the wave interaction through a discontinuous media provides information about vertical and in-plane variations of the SLD, which is related with the 3D structure and composition of the sample. Focusing on the specular reflectometry pattern, a "vertical" profile of the SLD is obtained and by a fitting model information about embedded interfaces, thickness of layers, roughness, vertical composition of layers, interlayer diffusion, etc. can be obtained. Analysis of off-specular reflectometry can provide information about in plane structures (composition, phase separation, magnetic domains, correlated roughness...) on both surface and buried interfaces. In this article we will focus on the specular neutron reflectometry signal.
Neutron reflectivity was measured on the instrument D17 at ILL [33]. The configuration of the reflectometer was the Time Of Flight (TOF) mode, with incident neutron wavelengths from $2 \AA$ to $25 \AA$ and with a $\mathrm{Q}$ range exploring $0.01 \AA^{-1}<\mathrm{Q}<0.1 \AA^{-1}$ at a fixed incident angle of $\theta=1.2^{\circ}$, which provides a resolution (wavelength dependent) of $0.5-1.2 \%$. The raw data was reduced using COSMOS [34].

The fits shown in all figures have been performed using "Motofit", a reflectivity analysis software package broadly used for NR data analysis developed by Andrew Nelson at ANSTO, Australia [35].

HAXPES measurements were carried out at the BM-25 CRG SpLine beamline at the ESRF, Grenoble, France [36,37]. The experimental set-up in which the experiment has been performed is devoted to HAXPES [38]. This experimental setup houses a UHV system and high electron kinetic energy analyzer, which is capable to work from few eV up to $15 \mathrm{keV}$, $[39,40]$. The detection geometry and consequently the counting rate can be optimized without changing neither the sample position nor the incoming beam impact point, i.e., without disturbing the diffraction geometry conditions, due to the electron analyzer is mounted on a 3-axis motorized table so that the analyzer focal point position can be adjusted on the sample.

HAXPES spectra were measured at $14 \mathrm{keV}, 11 \mathrm{keV}, 9 \mathrm{keV}$ and $7.5 \mathrm{keV}$ of photon energy, a pass energy of $200 \mathrm{eV}$ was used in order to measure the photoemission spectra in relatively short times. It should be mentioned that high energy resolution is essential for an electronic depth profile but not for a compositional depth profile. The compositional depth profile is obtained by following the intensity of the photoelectrons from the elements of the substrate and the overlayer film by changing the energy of the incident X-rays and hence the escape depth (kinetic energy) of the photoelectrons. The HAXPES experimental data have been interpreted considering that the photoemission intensity for photoelectrons is attenuated following the model explained in Annexure II.

\section{Results and discussion}

First, a control reference NR experiment was performed: an ITO film on top of glass was measured at room temperature $(300 \mathrm{~K})$, which allowed us to determine with precision the thickness of ITO $(135 \mathrm{~nm}$ with roughness $\sim 3 \mathrm{~nm}$ ) and to confirm the good in-plane homogeneity of the film (almost total absence of off-specular reflection) (Fig. 1).

The Scattering Length Density of ITO was previously calculated: $3.88 \times 10^{-6} \AA^{-2}$, the value was confirmed by a good fit (red line in Fig. 1) which also provided an experimental value for the thickness

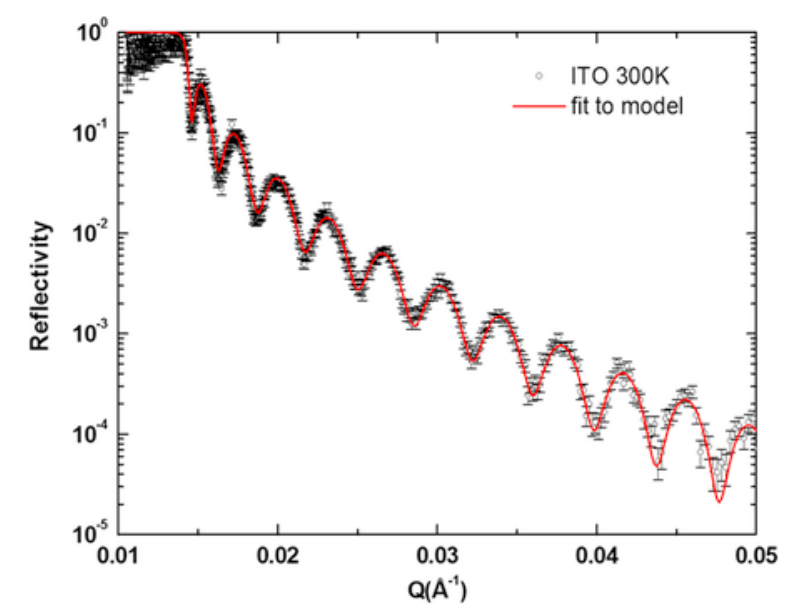

Fig. 1. Neutron reflectivity of an ITO film on top of silica fused glass (symbols). Fits to model (red line) delivered the SLD, thickness, and roughness of the ITO film. (For interpretation of the references to color in this figure legend, the reader is referred to the web version of this article.) 
and roughness of the ITO layer, respectively of $135 \mathrm{~nm}$ and $3 \mathrm{~nm}$. These three parameters: ITO scattering length density, thickness and roughness of the layer were kept fixed for the fitting of subsequent reflectometry plots.

The following NR and HAXPES experiments started with an initial measurement of a [P3HT:PCBM] [1.1:1] blend layer (active layer in a solar cell) cast on a glass/ITO substrate, at room temperature $(300 \mathrm{~K})$ the sample was then annealed increasing the temperature in steps until a new stable temperature was reached and a new scans were measured. For the HAXPES experiment, the in-situ annealing was performed in UHV. This sequential procedure presents an evolution of reflectometry and HAXPES spectra until the maximum temperature which was measured ( $415 \mathrm{~K})$.

Regarding NR, the fits of the experimental data were performed using Motofit (see Section 2 for methodological details); after several trials for each fitting procedure, the best fitting results were obtained assuming a structure of 20 additional "layers" within this blend placed on top of the previously determined glass/ITO layers whose parameters were not varied during the fitting process. This "structure" in 20 layers of the blend was kept for all fits and although the SLD were varied for all layers in all plot fittings, the width of each layer is kept fixed. When good convergence is obtained, SLD of each layer is recorded (imposing continuity and total thickness of sample as constraints for the fit), a SLD profile is obtained for the full [P3HT:PCBM] layer. Fig. 2 shows NR experimental data and the fit curves obtained at $300 \mathrm{~K}$ (left) and $415 \mathrm{~K}$ (right).

The temperature sequence $(360 \mathrm{~K}, 385 \mathrm{~K}, 415 \mathrm{~K}$ and finally cooling down back to $300 \mathrm{~K}$ ) for NR measurements is presented in a family of plots in Fig. 3, where every reflectometry spectra is compared to the original at $300 \mathrm{~K}$. It should to be noted that the values obtained for one temperature are used as a starting point for the fit of the subsequent temperature, until the final fit at $415 \mathrm{~K}$ is obtained. After the highest temperature is measured, the temperature was brought down to $300 \mathrm{~K}$ and a final measurement was taken in order to compare room temperature composition before and after the thermal annealing process (Fig. 3d). Finally, from a calculation of SLD of pure P3HT and PCBM a compositional profile can be extracted as a function of depth by linearly combining the contribution of the two materials to the measured "intermediate" SLD. The contrast in SLD between P3HT and PCBM is enough to apply this procedure even if none of the components of the blend are deuterated.

The sequential in situ thermal annealing creates an evolution of the reflectivity pattern of the samples with some features that are more or less unchanged, in contrast with other Q-ranges where stronger modification or appearance of additional maxima are occurring, in particular the new maximum at $Q=0.025 \AA^{-1}$, and in minor degree the onset of a maximum at $Q=0.015 \AA^{-1}$ and the increase in signal intensity for the higher $\mathrm{Q}$ values, in a region of the plot at which the fit is less accurate. From an analysis of the progress of the fitting runs, the two above mentioned new maxima can be associated with changes near the interfaces of the active layer with the ITO and air surfaces, while the more stable features correspond to the fixed glass/ITO sublayers plus the additional evolution of the bulk [P3HT:PCBM] constructed with the central "layers" of the fitting scheme. The result for the final $300 \mathrm{~K}$ measurement (after the annealing process) is very similar to the one at $415 \mathrm{~K}$ (Fig. 3d), demonstrating that the evolution of composition driven by the temperature is stabilized after the cooling process, showing a persistence of the internal structure created by the thermal annealing.

HAXPES measurements were performed on the active layer P3HT:PCBM (without the Al electrode) to confirm the depletion of P3HT near the surface induced by the annealing process. Since both components of the blend are organic compounds, it is a complex task to monitor the amount of P3HT or PCBM following the evolution of the carbon $\mathrm{C} 1 \mathrm{~s}$ peak as a function of the annealing temperature. For this reason, we have used the sulphur S 1 s peak as fingerprint to measure the amount of P3HT as compared to the amount of PCBM. Fig. 4 shows the evolution of S/O and S/C intensity ratios as function of air and vacuum annealing temperature, for a photon energy of $14 \mathrm{keV}$. The $\mathrm{S} / \mathrm{C}$ ratios, shown in Fig. 4 (blue and purple triangles) do not present significant variation with the annealing temperature since both materials have a strong carbon content. However, if we compare the S/O ratio a clear variation can be appreciated. The $\mathrm{S} / \mathrm{O}$ ratio at $300 \mathrm{~K}$ is around 28 , while for the annealed sample is only about 2 , which is a clear indication that the sulphur (P3HT) content at the surface decreases strongly with the annealing at $395 \mathrm{~K}$ in air, in agreement with composition profile obtained from the SLD shown in Fig. 5, at least for the depths probed by HAXPES at the photon energy of $14 \mathrm{keV}$, which is around $50 \mathrm{~nm}$.

In Fig. 5a superposition of the compositional profile (\% volume) of the [P3HT:PCBM] layer obtained from the fits is shown; it emphasizes the evolution between the initial $300 \mathrm{~K}$ profile and the $415 \mathrm{~K}$ profile, while it is kept stable during the cooling process, with strong similarity between the $415 \mathrm{~K}$ profile and the $300 \mathrm{~K}$ after annealing. The main feature of this evolution is the reduction of the initial PCBM content at the centre of the layer and the migration of the molecules to both interfaces, the one in contact to air and more strongly to the one in contact to ITO, where sub-interface composition reaches $80 \%$ volume of PCBM. Curiously, this evolution has similar features to the one reported by Kiel et al. $[30,31]$ for the air surface but not for the internal substrate/active layer interface. For the air surface, the highest PCBM content is
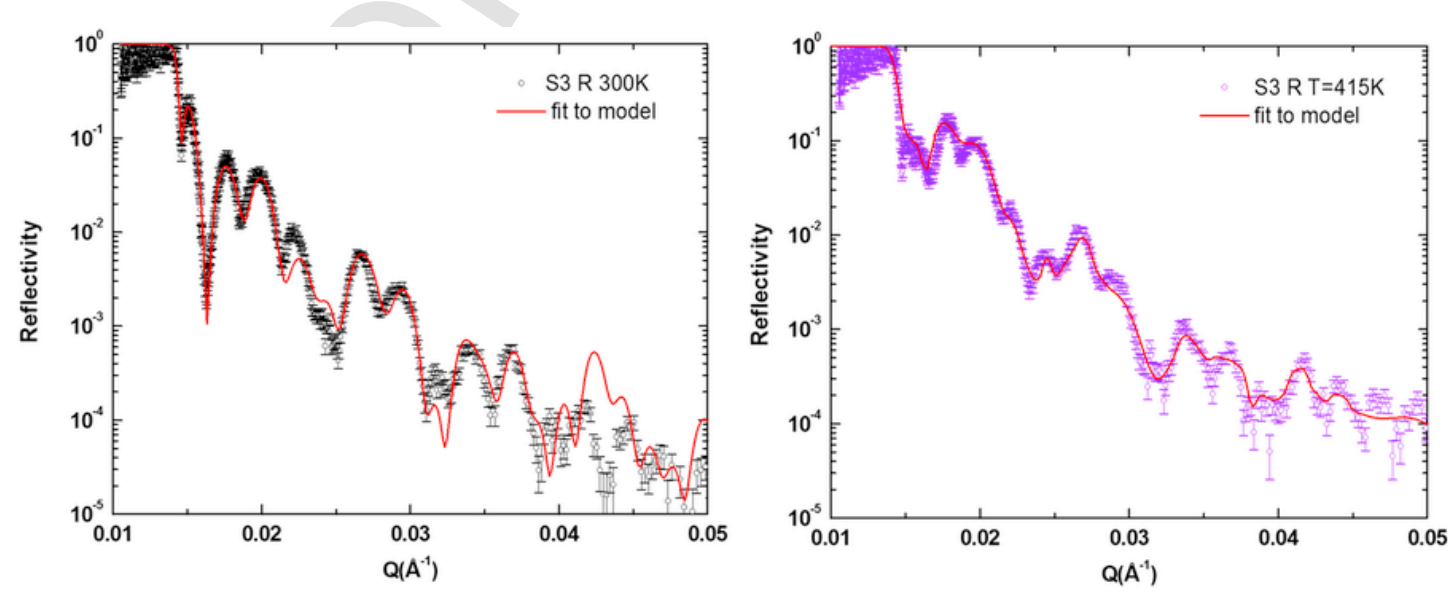

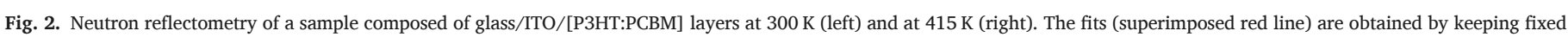

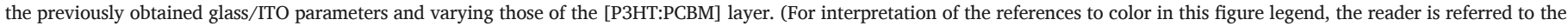
web version of this article.) 

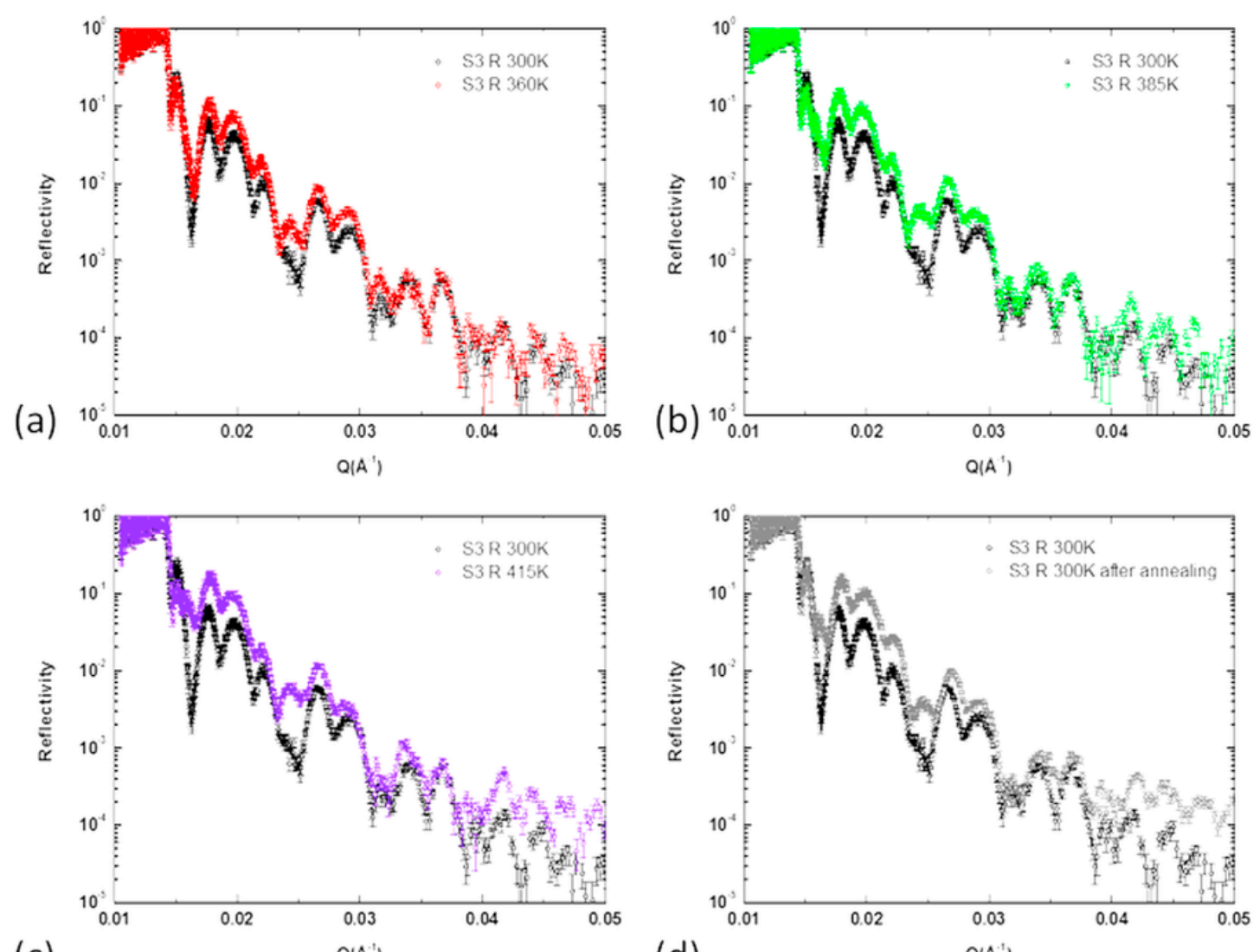

(c)

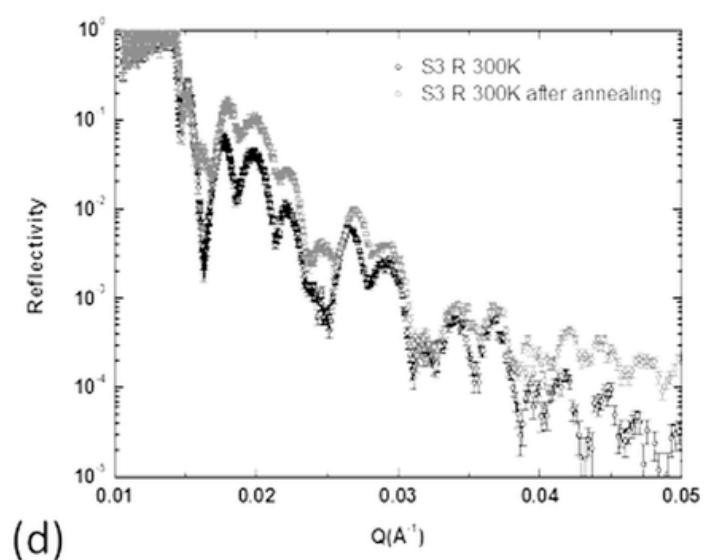

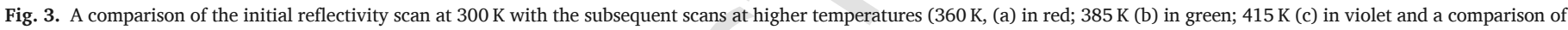

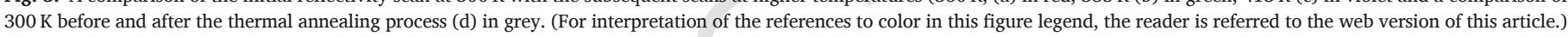
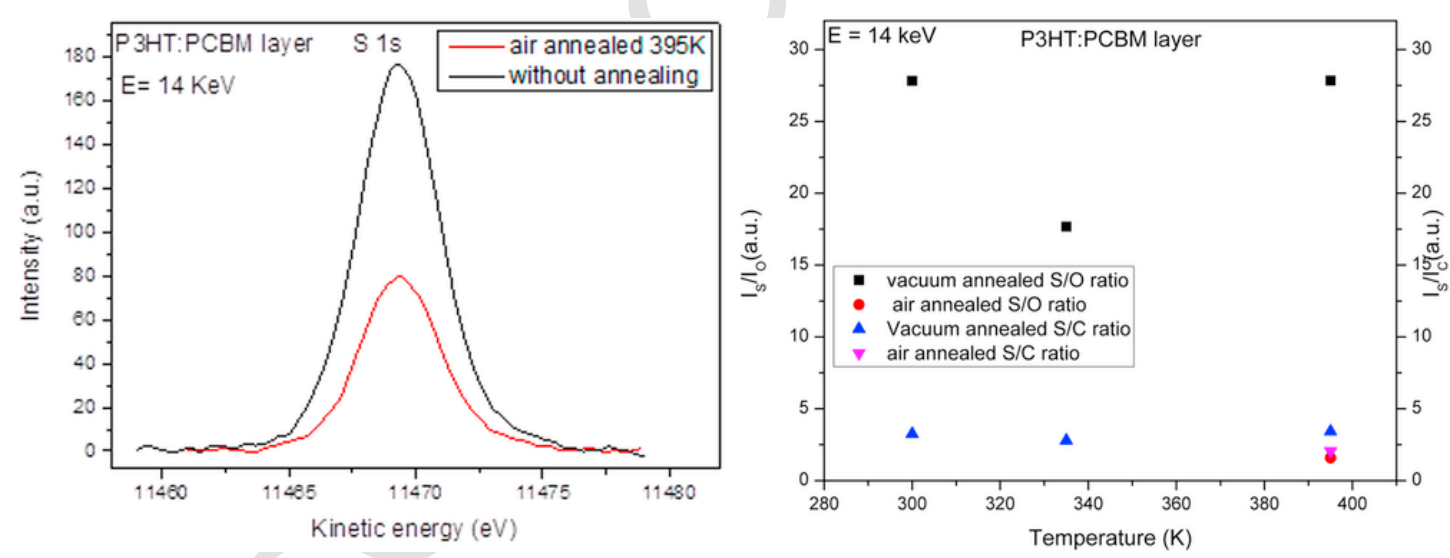

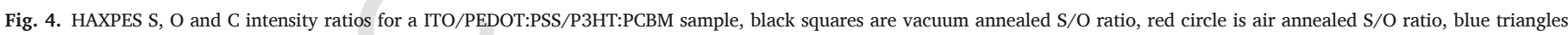

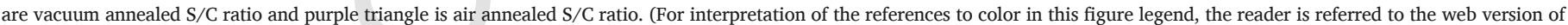
this article.)

found by Kiel in a sub-surface layer and not in the surface itself and also there are some differences, because the central composition in Kiel's work is kept constant. However, from our results shown in Fig. 5 it is evident that a reduction in PCBM content in the central part is obtained and it is the source of the molecules that are migrating to the areas where its concentration is increased upon annealing.

HAXPES experiments on the outer layers (for a depth around $50 \mathrm{~nm}$ for the maximum incident energy of $14 \mathrm{keV}$, as indicated by an arrow in Fig. 5) confirm the reduction of P3HT content near the surface upon annealing.
The HAXPES technique enables the study of buried inferfaces. For this reason, the samples with an Al top electrode around $38 \mathrm{~nm}$ thick can be measured. Again we use the $S$ 1s peak as a fingerprint of the P3HT content in the photoactive buried layer. The S 1S, Al 1s ratio for a complete device without annealing is about 0.09 (black square), while after annealing in air at $395 \mathrm{~K}$ this ratio decreases until 0.03 (red circle), as can be observed in Fig. 6. It is a clear indication that the P3HT amount at the Al/P3HT:PCBM interlayer decreases with the annealing temperature in air, similarly to the case without the Al electrode. 


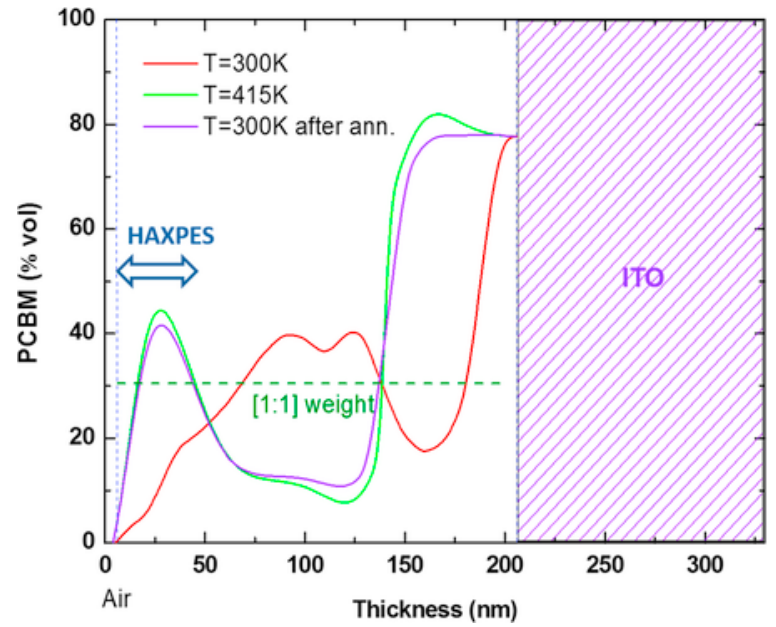

Fig. 5. Composition profile (\% volume of PCBM) obtained from the experimental scattering length density (SLD) for the sample at $300 \mathrm{~K}$ (red), $415 \mathrm{~K}$ (green) and again $300 \mathrm{~K}$ (blue) after the thermal annealing. The area explored by HAXPES is indicated by a double arrow. (For interpretation of the references to color in this figure legend, the reader is referred to the web version of this article.)

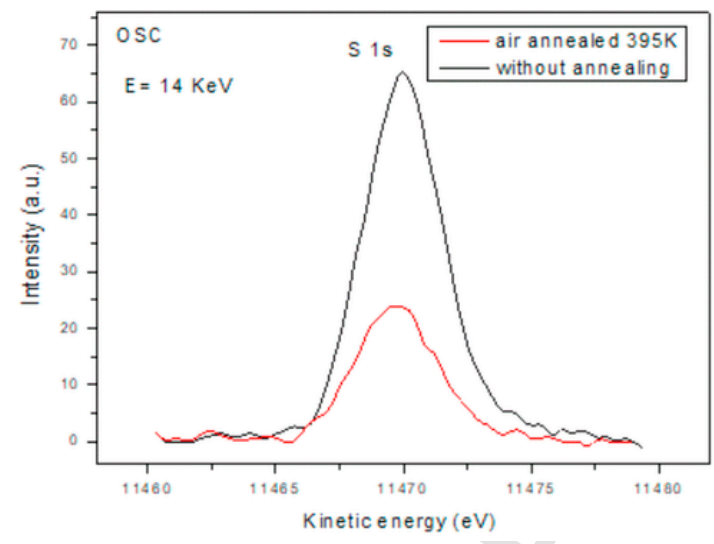

In order to follow in situ the compositional evolution near the top electrode, HAXPES spectra have been measured after annealing in UHV conditions in the same chamber at different photon energies $(7.5 \mathrm{keV}, 9$ $\mathrm{KeV}, 11 \mathrm{keV}$ and $14 \mathrm{keV}$ ). To model the depth profile (P3HT:PCBM composition) inside the device and following the method presented in Ref. [40], a linear inter-diffusion profile model at the interface between $\mathrm{Al}$ and the photoactive layer has been considered as a bilayer system where the $\mathrm{S} 1 \mathrm{~s}$ signal is the substrate signal and the $\mathrm{Al}$ 1s signal is the overlayer signal. This method allows us to represent the photoemission intensity as function of the electron kinetic energy, for three UHV temperatures $300 \mathrm{~K}, 335 \mathrm{~K}$ and $395 \mathrm{~K}$, respectively. In a linear inter-diffusion profile model only two parameters are free during the fitting procedure: the overlayer thickness and the diffusion width at the interface.

Fig. 7 shows the evolution of S 1 s intensity properly normalized as function of photon kinetic energy for two annealing temperatures ( $335 \mathrm{~K}$ and $395 \mathrm{~K}$ ) and room temperature. From a qualitative point of view the intensity of the $S 1$ s peak decreases with the annealing temperature providing an indication that the P3HT content decreases at the $\mathrm{Al}$ interface. The fittings are good considering the error bars of the experimental points (see Fig. 7 left), thus providing a result for the $\mathrm{Al}$ overlayer thickness of $38 \mathrm{~nm}$ for all temperatures, while for the diffusion width at room temperature the model provides a value of $16 \mathrm{~nm}$, which after the annealing at $335 \mathrm{~K}$ decreases until $13 \mathrm{~nm}$ and is maintained constant for $395 \mathrm{~K}$ of annealing temperature. Once these parameters have been obtained, the sulphur (P3HT) depth profile composi-

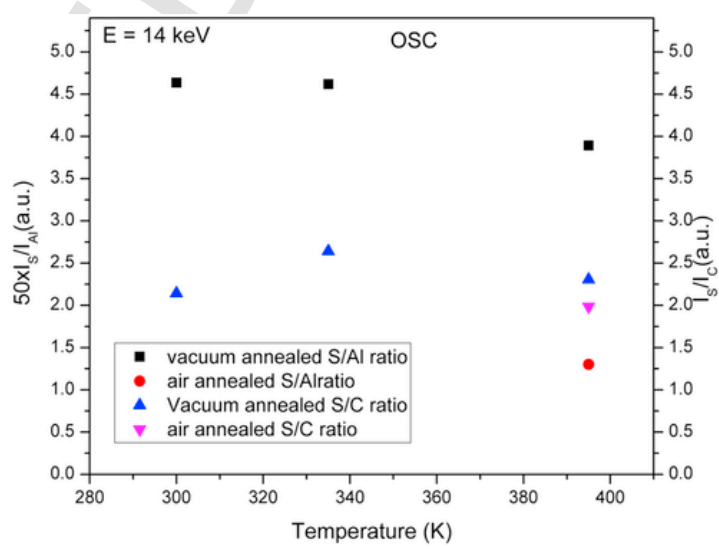

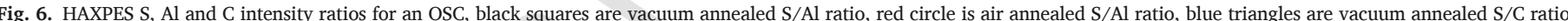

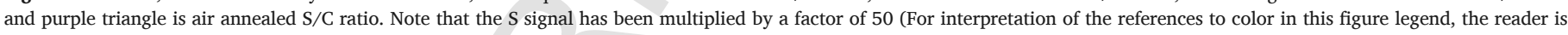
referred to the web version of this article.).
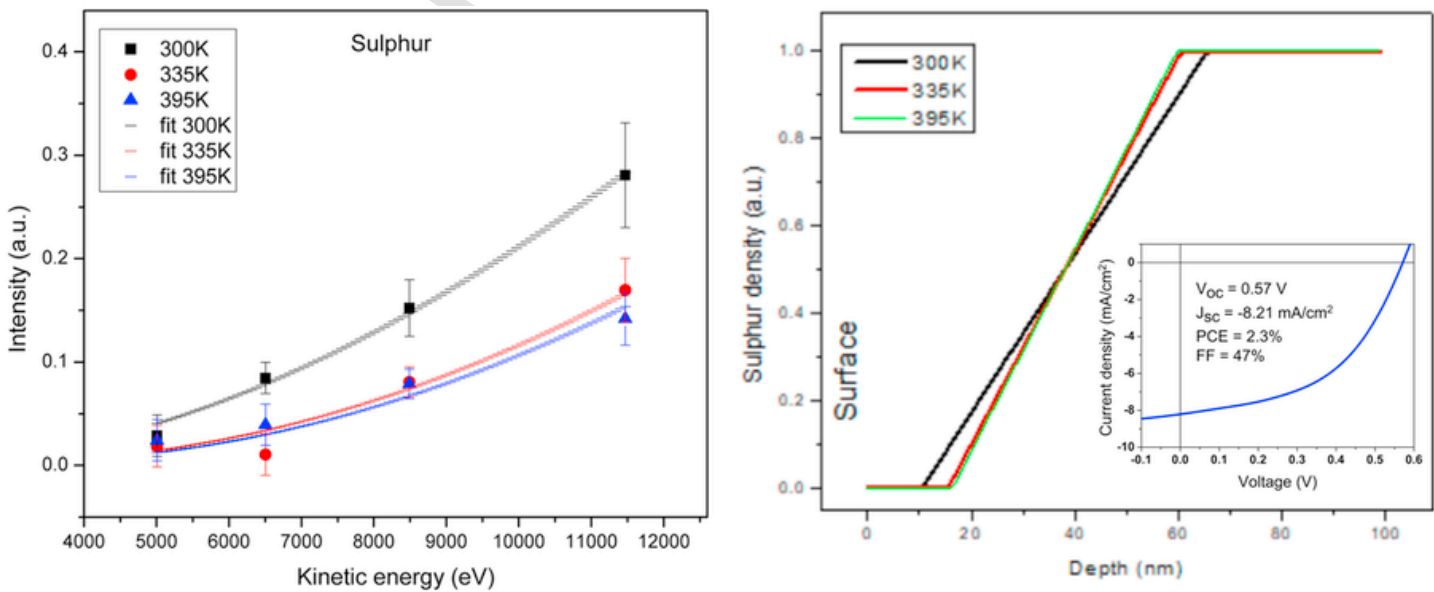

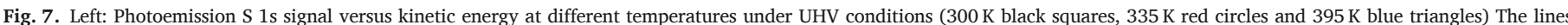

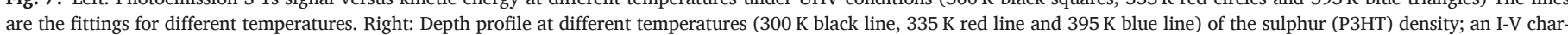
acteristic of a typical measured OSC is included as an insert. (For interpretation of the references to color in this figure legend, the reader is referred to the web version of this article.) 
tion can be modelled as shown in Fig. 7, confirming that after the UHV annealing the P3HT content at the $\mathrm{Al}$ interface decreases.

From an operational point of view of the solar cell it would be desirable that a composition profile with an increasing content of PCBM towards the $\mathrm{Al}$ electrode is obtained by the thermal annealing process in the blend which conforms the active layer of the solar cell. This profile is not fully achieved because although a convenient increase of PCBM content is obtained in the interface with top $\mathrm{Al}$ electrode, there is also a strong increase near the ITO interface, which is bad for charge transport (electron injection) in this electrode for a solar cell with standard architecture. But it should be considered that thermal annealing has been reported to increase solar cell power conversion efficiency because the mobility of charges is improved if the conjugated polymer is more crystalline, and it is well known that polymer crystallinity is improved by thermal annealing $[17,18]$. Neutron reflectometry cannot distinguish between the amorphous and crystalline phase (because they have very similar SLD) and therefore a measurement of the crystallinity evolution cannot be performed during the experiments in which the evolution of the concentration profile was measured. The balance between both effects is positive, but not from the side of the reorganization of the PCBM within the active layer. Additionally, this reorganization will not improve the charge collection for solar cells with inverted architecture, although the relatively higher increase in PCBM concentration near the ITO interface is better, in this case the accumulation of PCBM in the air (or future electrode required for inverted architecture) will be detrimental for charge extraction.

Globally, our result confirm that PCBM tends to increase its concentration at the surface upon annealing, as reported by Campoy [22] and Parnell [29]; also Kiel [30,31] pointed to an increase of PCBM content immediately below the surface but keeping a higher content of P3HT at the surface itself. On the other hand, Zhang [25] reported opposite observation with a higher crystallinity of P3HT preventing the increase in PCBM concentration near the surface. Our experiment indicates that although annealing has been used to improve polymer crystallinity in this case does not prevent PCBM from migrating to the outer parts of the active layer, and in particular to the air (or $\mathrm{Al}$ ) interface.

Since the evaporation of $\mathrm{Al}$ electrode prior to the annealing process seems to avoid a stronger redistribution of the PCBM content, it is recommended to proceed in this order: first, $\mathrm{Al}$ evaporation, then thermal annealing. In this way, the manufacturer keeps higher the concentration of PCBM in the bulk (central area, separated from the interfacial areas within the active layer) and avoids creating concentration "barriers" that impedes efficient charge extraction to the $\mathrm{Al}$ or ITO electrodes for standard or inverted solar cells respectively, while the beneficial effects of thermal annealing via an increase in crystallinity is preserved. The findings provide a better understanding of the interplay between the manufacturing sequence (thermal annealing and layer deposition or evaporation) and the final internal structure of the active layer. Depending on the architecture of the final device, a different order is recommended in order to obtain better composition profiles for charge collection and transfer to the electrodes: annealing after top electrode deposition for standard cells, annealing before top electrode deposition for inverted cells.

\section{Conclusions}

The evolution of the vertical composition of a [P3HT:PCBM][1.1:1] active layer of solar cells has been measured by Neutron Reflectometry on D17 at ILL and by HAXPES at the BM-25 beamline at ESRF. The measurements are performed on a set of samples that reproduce the manufacturing steps of a functional solar cell, which are the sequential deposition of ITO, then the P3HT:PCBM blend and finally a top Al electrode on a fused silica substrate and a thermal annealing cycle. Our measurements are focused on this final annealing step, and data has been measured before and after the evaporation of the top Al electrode.

The SLD contrast between the conjugated polymer P3HT and the fullerene derivative PCBM which comprises the active layer is enough to obtain a composition profile from the experimental SLD variation obtained by fitting the neutron reflectometry data to a simple model based on a sequence of layers. The SLD and roughness of ITO on glass $\left(\right.$ SLD $($ ITO $)=3.88 \times 10^{-6} \AA^{-2}$; thickness $135 \mathrm{~nm}$ and roughness $3 \mathrm{~nm}$ ) have been measured and the obtained parameters are kept as fixed parameters for subsequent fitting of more complex reflectometry spectra. The calculated SLDs of P3HT $\left(6.72 \times 10^{-7} \AA^{-2}\right)$ and PCBM $\left(4.34 \times 10^{-6}\right.$ $\AA^{-2}$ ) have been used to match the SLD profile obtained by fitting the experimental spectra and therefore obtaining a composition profile (in \% volume) for all the thermal annealing steps from $300 \mathrm{~K}$ to $415 \mathrm{~K}$. After reaching this maximum temperature and cooling down to $300 \mathrm{~K}$, a very similar profile to the one at higher temperature is obtained, therefore the system is considered stable after annealing. This profile reduces the initial concentration of PCBM in the central part of the active layer and increases its concentration towards both interfaces, reaching the maximum content (around 80\% volume) in the interface with ITO and with a lower increase (up to $40 \%$ volume) near the air surface (or more exactly sub-surface interface, because at the interface itself the PCBM content tends to zero). The obtained composition at the ITO interface is better for inverted architecture and bad for standard architecture; at the air interface the effect is opposite but with less impact. If the annealing process is performed after evaporation of the top $\mathrm{Al}$ electrode, the effect of reducing the P3HT content near the electrode is confirmed by HAXPES measurements which shows a decrease of P3HT (and therefore an increase of PCBM concentration) near the $\mathrm{Al}$ electrode. This result provides a manufacturing recommendation about the sequence of annealing/deposition of final electrode depending on the architecture of the device: for standard devices it is better to first deposit the electrode and then thermally anneal the device; while the opposite is true for the inverted architecture. The additional benefits of thermal annealing via improved crystallinity of the conjugated polymer will be preserved in both cases.

\section{Uncited reference}

\section{[42].}

\section{Acknowledgements}

Helpful comments are acknowledged to Andrew Nelson, developer of the "Motofit" software package. This work was supported by Project ENE2016-79282-C5-5-R funded by MINECO-Spain (including FEDER funds) and Project 19882-GERM-15 funded by Fundación Séneca (Región de Murcia, Spain). We acknowledge Institute Laue Langevin (ILL) for beamtime allocation at D17, and Instituto de Ciencia de Materiales de Madrid (ICMM-CSIC) and European Synchrotron Radiation Facility (ESRF) for beamtime allocation at line BM-25.

\section{Annexure I}

Neutron reflectivity is an experimental technique which benefits from the wave properties of the neutron that can be expressed as a quantum mechanical wave, solution of the Schrödinger equation in a medium:

$\Psi \quad(\boldsymbol{r})=e^{i \boldsymbol{k} \bullet \boldsymbol{r}}$

which in vacuum is expressed with a wavenumber $\mathrm{k}$ given by:

$$
k^{2}=k_{0}^{2}=\frac{2 m E}{\mathrm{p}^{2}}
$$

with the neutron mass $m$, its energy $E$ and Planck's constant $\hbar$. Inside 
the material of potential $\boldsymbol{V}, k$ takes the value:

$k^{2}=\frac{2 m(E-V)}{\mathrm{p}^{2}}=k_{0}^{2}-4 \pi \rho$

and a refractive index " $n$ " can be defined as:

$k / k_{0}=n$

and approximated by:

$n=1-\frac{\lambda^{2} \rho}{2 \pi}$

Where $\lambda$ is the neutron wavelength and, $\rho$ is the nuclear "scattering length density" (SLD) which is related to the nucleus-neutron interaction potential (Fermi potential):

$\boldsymbol{V}(\boldsymbol{r})=\frac{2 \pi \mathrm{p}^{2}}{m} b \delta(\boldsymbol{r})$

with the isotope dependent scattering length $b$. To calculate the SLD and the final potential inside the medium one has to sum up all isotopes composing a molecule and normalize by its volume:

$\rho=\frac{1}{\text { volume }} \sum_{i} b_{i}$

$V=\frac{2 \pi \mathrm{p}^{2}}{m} \rho$

Those can be calculated using empirical values for the scattering lengths $\left(b_{i}\right)$ and number densities [41].

Annexure II

HAXPES experimental data have been interpreted considering that the photoemission intensity for photoelectrons of a q-ionization-subshell produced from a constituent element $A$ located between depth $\mathrm{Z}_{\mathrm{A}}$ and $\mathrm{Z}_{\mathrm{B}}$ of a laterally $(\mathrm{x}, \mathrm{y})$ homogeneous sample, within the analyzed area, is proportional to [42]:

$I_{A q}\left(h \vartheta, E_{\text {Kin }}\right) \propto \int_{\mathrm{ZA}}^{\mathrm{ZB}} n_{A}(z) \exp \left(\frac{-z}{E A L\left(E_{\text {Kin }}\right) \cos \theta}\right) d z$

Where EAL is the effective attenuation length of the photoelectrons, $\mathrm{n}_{\mathrm{A}}(\mathrm{z})$ is the density profile with depth and $\theta$ denotes the angle between the collection direction and the surface normal. Therefore we can determine the concentration depth profile $\mathrm{n}_{\mathrm{A}}(\mathrm{z})$ modeling the dependence of the photoemission intensity with the EAL using a linear profile model from references $[40,43]$. The maximum X-ray incident energy of $14 \mathrm{keV}$ provides a kinetic energy of photoelectrons of $13.5 \mathrm{keV}$, probing a maximum depth into the sample of $\sim 55 \mathrm{~nm}$.

\section{References}

[1] J.H. Burroughes, D.D.C. Bradley, A.R. Brown, R.N. Marks, K. Mackay, R.H. Friend, P.L. Burns, A.B. Holmes, Light-emitting diodes based on conjugated polymers, Nature 347 (1990) 539, https://doi.org/10.1038/347539a0.

[2] European Photovoltaic Technology Platform, A Strategic Research Agenda for Photovoltaic Solar Energy Technology, ISBN 978-92-79-05523-2, 2007.

[3] C.J. Brabec, N.S. Sariciftci, J.C. Hummelen,"Plastic Solar Cells", Adv. Funct. Mater 11 (2001) 15, https://doi.org/10.1002/1616-3028(200102).

[4] H. Sirringhaus, Device Physics of Solution-Processed Organic Field-Effect Transistors, Adv. Mater. 17 (2005) 2411, https://doi.org/10.1002/adma.200501152.

[5] Gilles Dennler, MarkusC. Scharber, Christoph J. Brabec, Polymer-Fullerene Bulk-Heterojunction Solar Cells, Adv. Mater. 21 (2009) 1323-1338, https://doi. org/10.1002/adma.200801283.

[6] Amrita Haldar, Kang-Shyang Liao, Seamus A. Curran, Effect of printing parameters and annealing on organic photovoltaics performance, J. Mater. Res. 27 (2012) 2079-2087, https://doi.org/10.1557/jmr.2012.217.
[7] Jingbi You, Letian Dou, Ken Yoshimura, Takehito Kato, Kenichiro Ohya, Tom Moriarty, Keith Emery, Chun-Chao Chen, Jing Gao, Gang Li, Yang Yang, "A polymer tandem solar cell with $10.6 \%$ power conversion efficiency", Nat. Commun. 4 (2013) 1446, (:1446 10.1038/ncomms2411).

[8] Yuhang Liu, Jingbo Zhao, Zhengke Li, Cheng Mu, Wei Ma, Huawei Hu, Kui Jiang, Haoran Lin, Harald Ade, He Yan, Aggregation and morphology control enables multiple cases of high-efficiency polymer solar cells Yuhang, Nat. Commun. 5 (2014) 5293, https://doi.org/10.1038/ncomms6293.

[9] Jingbo Zhao, Yunke Li, Guofang Yang, Kui Jiang, Haoran Lin, Harald Ade, Wei Ma, He Yan,"Efficient organic solar cells processed from hydrocarbon solvents", Nat. Energy 1 (2016) 15027, https://doi.org/10.1038/NENERGY.2015.27.

[10] Cenqi Yan, Stephen Barlow, Zhaohui Wang, He Yan, Alex K.-Y. Jen, Seth R. Marder, Xiaowei Zhan, Non-fullerene acceptors for organic solar cells, Nat. Rev. Mater. 3 (2018) 18003, (doi:0.1038/natrevmats.2018.3).

[11] Frederik C. Krebs, Suren A. Gevorgyan, Jan Alstrup, A roll-to-roll process to flexible polymer solar cells: model studies, manufacture and operational stability studies, J. Mater. Chem. 19 (2009) 5442, https://doi.org/10.1039/B823001C.

[12] Frederik C. Krebs, All solution roll-to-roll processed polymer solar cells free from indium-tin-oxide and vacuum coating steps, Org. Electron. 10 (2009) 761-768, https://doi.org/10.1016/j.orgel.2009.03.009.

[13] S.K. Hau, H.L. Yip, A.K.Y. Jen, A review on the development of the inverted polymer solar cell architecture, Polym. Rev. 50 (2010) 474-510, https://doi.org/10. 1080/15583724.2010.515764.

[14] M. Jørgensen K. Norrman S.A. Gevorgyan T. Tromholt B. Andreasen F.C. Krebs Stability of polymer solar cells Adv. Mater. 24 (2012) 580-612 https://doi.org/10 1080/15583724.2010.515764

[15] Jian Ye, Xueliang Li, Jianjun Zhao, Xuelan Mei, Qian Li, Solution-processed P3HT-functional graphene for efficient heterojunction organic photoelectronics, J. Mater. Res. 31 (2016) 1985-1992, https://doi.org/10.1557/jmr.2016.243.

[16] Nieves Espinosa, Rafael García-Valverde, Antonio Urbina, Frederik C. Krebs, A life cycle analysis of polymer solar cell modules prepared using roll-to-roll methods under ambient conditions, Sol. Energy Mater. Sol. Cells 95 (2011) 1293, https://doi. org/10.1016/j.solmat.2010.08.020.

[17] T. Agostinelli, S. Lilliu, J.G. Labram, M. Campoy Quiles, M. Hampton, E. Pires, J. Rawle, O. Bikondoa, D.D.C. Bradley, T.D. Anthopoulos, J. Nelson, J.E. Macdonald, Real time investigation of crystallization and phase segregation dynamics in P3HT:PCBM solar cells during thermal annealing, Adv. Funct. Mater. 21 (2011) 1701-1708, https://doi.org/10.1002/adfm.201002076.

[18] Jose Abad, Nieves Espinosa, Pilar Ferrer, Rafael García-Valverde, Carmen Miguel, Javier Padilla, Alberto Alcolea, German R. Castro, Jaime Colchero, Antonio Urbina, Molecular structure of poly(3-alkyl-thiophenes) investigated by calorimetry and grazing incidence X-ray scattering, Sol. Energy Mater. Sol. Cells 97 (2012) 109-118, https://doi.org/10.1016/j.solmat.2011.09.025.

[19] Carlos Díaz-Paniagua, Antonio Urbina, Victoria García-Sakai, Tilo Seydel, José Abad, Javier Padilla, Rafael García-Valverde, Nieves Espinosa, María-José Gómez-Escalonilla, Fernando Langa, Francisco Batallán, Molecular dynamics of solutions of poly-3-octyl-thiophene and functionalized single wall carbon nanotubes studied by neutrón scattering, J. Chem. Phys. 427 (2013) 129, https://doi.org/10. 1016/j.chemphys.2013.06.016.

[20] Anne A.Y. Guilbert, Antonio Urbina, Jose Abad, Carlos Díaz-Paniagua, Francisco Batallán, Tilo Seydel, Mohamed Zbiri, Victoria García-Sakai, Jenny Nelson, Temperature-dependent dynamics of polyalkylthiophene conjugated polymers: a combined neutron scattering and simulation study, Chem. Mater. 27 (2015) 7652, https://doi.org/10.1021/acs.chemmater.5b03001.

[21] Cecilia M. Bjorström1, Andrzej Bernasik, Jakub Rysz, Andrzej Budkowski, Svante Nilsson, Mattias Svensson, Mats R. Andersson, Kjell O. Magnusson, Ellen Moons, Multilayer formation in spin-coated thin films of low-bandgap polyfluorene:PCBM blends, J. Phys.: Condens. Matter 17 (2005) L529-L534, https://doi.org/10.1088/ 0953-8984/17/50/L01.

[22] Mariano Campoy-Quiles, Toby Ferenczi, Tiziano Agostinelli, Pablo G. Etchegoin, Youngkyoo Kim, Thomas D. Anthopoulos, Paul N. Stavrinou, Donal D.C. Bradley, Jenny Nelson, Morphology evolution via self-organization and lateral and vertical diffusion in polymer: fullerene solar cell blends, Nat. Mater. 7 (2008) 158, https:// doi.org/10.1038/nmat2102

[23] David S. Germack, Calvin K. Chan, R. Joseph Kline, Daniel A. Fischer, David J. Gundlach, Michael F. Toney, Lee J. Richter, Dean M. DeLongchamp, Interfacial segregation in polymer/fullerene blend films for photovoltaic devices, Macromolecules 43 (2010) 3828-3836, https://doi.org/10.1021/ma100027b.

[24] P.G. Karagiannidis, D. Georgiou, C. Pitsalidis, A. Laskarakis, S. Logothetidis, Evolution of vertical phase separation in P3HT:PCBM thin films induced by thermal annealing, Mater. Chem. Phys. 129 (2011) 1207-1213, https://doi.org/10.1016/j. matchemphys.2011.06.007.

[25] C.M. Zhang, Y.F. Hu, A.W. Tang, Y. Ning, L.F. Lv, F. Teng, The effect of post-annealing on the interface between the aluminum electrode and the active layer in polymer/fullerene solar cells, Appl. Phys. A 117 (2014) 1335-1341, https://doi. org/10.1007/s00339-014-8550-9.

[26] Zhengguo Xiao, Yongbo Yuan, Bin Yang, Jeremy VanDerslice, Jihua Chen, Ondrej Dyck, Gerd Duscher, Jinsong Huang, Universal formation of compositionally graded bulk heterojunction for efficiency enhancement in organic photovoltaics, Adv. Mater. 26 (2014) 3068-3075, https://doi.org/10.1002/adma.201305196.

[27] Zheng Xu, Li-Min Chen, Guanwen Yang, Chun-Hao Huang, Jianhui Hou, Yue Wu, Gang Li, Chain-Shu Hsu, Yang Yang, Vertical phase separation in Poly(3-hexylthiophene): fullerene derivative blends and its advantage for inverted structure solar cells, Adv. Funct. Mater. 19 (2009) 1227-1234, https://doi.org/10.1002/adfm. 200801286. 
[28] M. Kaur, A. Gopal, R.M. Davis, J.R. Heflin, Concentration gradient P3OT/PCBM photovoltaic devices fabricated by thermal interdiffusion of separately spin-cast organic layers, Sol. Energy Mater. Sol. Cells 93 (2009) 1779-1784, https://doi.org/ 10.1016/j.solmat.2009.06.009.

[29] Andrew J. Parnell, Alan D.F. Dunbar, Andrew J. Pearson, Paul A. Staniec, Andrew J.C. Dennison, Hiroshi Hamamatsu, Maximilian W.A. Skoda, David G. Lidzey, Richard A.L. Jones, Depletion of PCBM at the cathode interface in P3HT/PCBM thin films as quantified via neutron reflectivity measurements, Adv. Mater. 22 (2010) 2444, https://doi.org/10.1002/adma.200903971.

[30] Jonathan W. Kiel, Brian J. Kirby, Charles F. Majkrzak, Brian B. Maranville, Michael E. Mackay, Nanoparticle concentration profile in polymer-based solar cells, Soft Matter 6 (2010) 641, https://doi.org/10.1039/B920979D.

[31] J.W. Kiel, M.E. Mackay, B.J. Kirby, B.B. Maranville, C.F. Majkrzak, Phase-sensitive neutron reflectometry measurements applied in the study of photovoltaic films, J. Phys. Chem. 133 (2010) 074902, https://doi.org/10.1063/1.3471583.

[32] James W. Kingsley, Pier Paolo Marchisio, Hunan Yi, Ahmed Iraqi, Christy J. Kinane, Sean Langridge, Richard L. Thompson, Ashley J. Cadby, Andrew J. Pearson, David G. Lidzey, Richard A.L. Jones, Andrew J. Parnell, Molecular weight dependent vertical composition profiles of PCDTBT:PC71BM blends for organic photovoltaics, Sci. Rep. 4 (2014) 5286, https://doi.org/10.1038/srep05286.

[33] Thomas Saerbeck, Robert Cubitt, Andrew Wildes Giuliana Manzin, Ken H. Andersen, Philipp Gutfreund, Recent upgrades of the neutron reflectometer D17 at ILL, J. Appl. Cryst. 51 (2018) 249-256, https://doi.org/10.1107/S160057671800239X.

[34] Philipp Gutfreund, Thomas Saerbeck, Miguel A. Gonzalez, Eric Pellegrini, Mark Laver, Charles Dewhurst and Robert Cubitt Towards generalized data reduction on a chopper-based time-of-flight neutron reflectometer, J. Appl. Cryst. 51 (2018) 606-615, https://doi.org/10.1107/S160057671800448X.

[35] Nelson Andrew, Co-refinement of multiple-contrast neutron/X-ray reflectivity data using MOTOFIT, J. Appl. Crystallogr. 39 (2006) 273, https://doi.org/10.1107/ S0021889806005073.
[36] J. Rubio-Zuazo, G.R. Castro, Probing buried interfaces by simultaneous combination of X-ray diffraction (SXRD) and hard X-ray photoelectron spectroscopy (HAX PES, up to $15 \mathrm{keV}$ ), Rev. Adv. Mater. Sci. 15 (2007) 79-86.

[37] G.R. Castro, Optical design of the general-purpose Spanish X-ray beamline for absorption and diffraction, J. Synchrotron Radiat. 5 (1998) 657, https://doi.org/10 1107/S0909049597019079.

[38] J. Rubio-Zuazo, G.R. Castro, Hard X-ray photoelectron spectroscopy (HAXPES) (p15 keV) at spline, the Spanish CRG beamline at the ESRF, Nucl. Instrum. Methods Phys. Res. A 547 (2005) 64-72, https://doi.org/10.1016/j.nima.2005.05.013.

[39] J. Rubio-Zuazo, G.R. Castro, Non-destructive compositional depth profile analysis by hard x-ray photoelectron spectroscopy, J. Phys. Conf. Ser. 100 (2008) 012042, https://doi.org/10.1088/1742-6596/100/1/012042.

[40] J. Rubio-Zuazo, P. Ferrer, G.R. Castro, Non-destructive compositional depth profile in the tens-of-nanometer scale, J. Electron Spectrosc. Relat. Phenom. 180 (2010) 27-33, https://doi.org/10.1016/j.elspec.2010.03.013.

[41] Neutron scattering lengths and cross sections at National Institute for Standards and Technology (NIST)-Center for Neutron Research: 〈http://www.ncnr.nist.gov/ resources/n-lengths/ $\rangle$, with data taken from Neutron News, 3, 29-37, 1992.

[42] J. Rubio-Zuazo, M. Escher, M. Merkel, G.R. Castro, High voltage-cylinder sector analyzer 300/15: a cylindrical sector analyzer for electron kinetic energies up to $15 \mathrm{keV}$, Rev. Sci. Instrum. 81 (2010) 043304, https://doi.org/10.1063/1.3398441.

[43] J. Rubio-Zuazo, M. Escher, N. Weber, M. Merkel, G.R. Castro, A two-dimensional event-counting detector for HV-CSA electrostatic analyzer at SpLine the Spanish CRG beamline at the ESRF, J. Phys.: Conf. Ser. 425 (2013) 082001, https://doi. org/10.1088/1742-6596/425/8/082001Highlights. 